\title{
Factors associated with persistent hypertension after puerperium among women with pre- eclampsia/eclampsia in Mulago hospital, Uganda
}

\author{
Emmanuel B Ndayambagye, Miriam Nakalembe, Dan K Kaye*
}

\begin{abstract}
Background: Women with severe pre-eclampsia/eclampsia are at risk of developing chronic hypertension in future. Chronic hypertension may manifest initially as persistent hypertension at the end of the puerperium. The objective was to determine the incidence and maternal biochemical, hematological and socio-demographic risk factors for persistent hypertension in patients with pre-eclampsia/eclampsia.

Methods: This was a prospective cohort study conducted from November 2008 to May 2009 at Mulago hospital labor ward and postnatal clinic. Participants were 200 women managed for pre-eclampsia/eclampsia and followed up to the end of the puerperium. Data was collected through using pre-coded interviewer-administered questionnaires, checking medical records and laboratory investigations. STATA (release 9) software was used for data analysis. At bivariate analysis, the relative risk of persistent hypertension was estimated the $95 \%$ confidence level. Using multivariate logistic regression analysis, factors that were independently associated with persistent hypertension were evaluated.
\end{abstract}

Results: Fifty four (27.7\%) out of the total 195 women had persistent hypertension after puerperium. Serum creatinine and the age of the patient were the only factors associated with persistence of hypertension after puerperium.

Conclusion: Nearly every one in four mothers with pre-eclampsia/eclampsia are at risk of persistent hypertension after the puerperium. Serum creatinine, serum uric acid and participants' age were the only factors independently associated with persistence of hypertension after the puerperium.

\section{Background}

Pre-eclampsia is an idiopathic multi-system disorder specific to pregnancy. It is characterized by hypertension (a blood pressure of higher of $140 / 90 \mathrm{mmHg}$ or higher in a previously normotensive women) and proteinuria. Pre-eclampsia and eclampsia complicate about 6-8\% all pregnancies worldwide [1] and are associated with increased maternal morbidity and mortality. They are also associated with development of chronic hypertension [2] as well as cardiovascular and renal complications later in life. Women with pre-eclampsia/eclampsia are at higher risk of obstetric complications like abruptio placenta and intra-uterine growth restriction [2] as

\footnotetext{
* Correspondence: dankkaye@yahoo.com

Department of Obstetrics and Gynecology, Makerere University Medical School, PO Box 7072, Kampala, Uganda
}

compared to normotensive women. They also have a higher risk of developing chronic hypertension in the future (14.8\% versus. $5.6 \%)$, and this risk increases markedly on long-term follow up (to $51 \%$ versus $14 \%$ ) [2].

Because they are at risk of developing chronic hypertension, all women with pre-eclampsia/eclampsia require follow up after delivery. Factors that predict chronic hypertension include maternal age and gestation age of onset of pre-eclampsia [3]. A higher maternal age [4] and lower gestation age at onset increase risk of chronic hypertension. During the postnatal period, the hypertension and proteinuria due to pre-eclampsia/eclampsia resolve within six weeks, and women having persistent hypertension and proteinuria thereafter may have an underlying cause. Postnatal review at 6-12 weeks after delivery provides an opportunity to ensure that manifestations of pre-eclampsia and any associated systemic 
complications have resolved. Early identification of chronic hypertension among these women during follow-up might prevent or reduce its long-term complications if relevant interventions to decrease or avert associated renal or cardiovascular damage are instituted in time. In Mulago hospital, about 3-4 patients with pre-eclampsia are seen per day, constituting $8.2 \%$ of all labour ward admissions. The objective of the study was to evaluate the incidence and factors associated with persistent hypertension in patients that present with pre-eclampsia/eclampsia.

\section{Methods}

Being Uganda's national referral hospital, Mulago receives women booked with the hospital's antenatal clinic, self referrals and referrals from other health units. The hospital conducts about 50-65 deliveries daily, over 25,000 deliveries per year. This was a prospective cohort study carried out in new Mulago hospital's labor ward and postnatal clinic, from $18^{\text {th }}$ November 2008 to $17^{\text {th }}$ March 2009. Participants were women admitted in New Mulago hospital with pre-eclampsia or eclampsia. These were followed up to the end of the puerperium. Hypertension in pregnancy was defined as any of the following: i) a blood pressure measurement of higher than or equal to $140 \mathrm{mmHg}$ systolic or $90 \mathrm{mmHg}$ diastolic (measured at rest on at least 2 occasions in a patient of more than 20 weeks of gestation); ii) a rise in systolic pressure of $30 \mathrm{mmHg}$ or higher and diastolic pressure of $15 \mathrm{mmHg}$ or higher, ((from the pre-pregnancy or first trimester measurement, measured on at least 2 occasions 4 hours apart,). All women with essential hypertension, chronic hypertension, hypertension in a prior pregnancy, a history of hypertension in the first trimester history, renal disease or pre- eclampsia in a prior pregnancy were excluded from the analysis.

Pre-eclampsia was defined as presence of hypertension (as defined above) and albuminuria (on urinary dipstick examination). A diagnosis of mild pre-eclampsia was made when a blood pressure of less than 160/110 $\mathrm{mmHg}$ was associated with proteinuria (albuminuria) of $2+$ or less on urinary dipstick examination, at any time during the management and subsequent follow up. Severe pre-eclampsia was diagnosed as a blood pressure of $160 / 110 \mathrm{mmHg}$ or higher in association with proteinuria of $3+$ or more on urinary dipstick examination, at any time during the management and subsequent follow up. A diagnosis of eclampsia was made when the patient with features of pre-eclampsia developed convulsions (tonic-clonic seizures). A diagnosis of HELLP syndrome was made where the patient presented with hypertension, elevated hepatic lactate dehydrogenase enzymes (greater than 600 units/liter), elevated aspartate and alanine transaminase enzymes (above 50 international units per liter) and platelet count less than 150,000 cells per mm3. (All patients with HELLP syndrome had features of severe pre-eclampsia. Presence of microangiopathic hemolytic anemia was not evaluated). The diagnosis of persistent hypertension was made when hypertension (as defined above) was found at any time six weeks or later postpartum.

\section{Data collection}

Data was collected through interviews using an interviewer-administered questionnaire. Other data was collected by participant examination, biomedical investigations and review of participants' medical records. This data included social-demographic factors (age, parity, marital status, spouse's age,); obstetric factors (such as gestational age at presentation, gestation age at disease onset, family history of hypertension or pre-eclampsia and severity of pre-eclampsia. Biochemical data included serial uric acid levels, creatinine levels, platelet count, urinary protein levels and results of renal function tests). Blood pressure was measured using the manual sphygmomanometer machine in a sitting position. Urine protein was determined by dipstick examination. The primary outcome was persistence of hypertension at six weeks postpartum. The sample size of 195 was estimated using the Kish and Leslie formula (1965) where $\mathrm{N}=\mathrm{Z}^{2} \mathrm{P}(1-\mathrm{P}) / \mathrm{D},{ }^{2}$ and assuming that the incidence of chronic hypertension on a follow up of women with pre-eclampsia/eclampsia is about 14.8\% [2].

All participants received antihypertensive and magnesium sulphate therapy according to a standard protocol as follows,: Magnesium sulphate $10 \mathrm{~g}$ intramuscularly together with $4 \mathrm{~g}$ intravenously were used as loading dose and $5 \mathrm{~g}$ intramuscularly every 4 hours until 24 hours later or as maintenance dose for all patients with severe pre-eclampsia and eclampsia. All patients with diastolic pressure above $100 \mathrm{mmHg}$ received hydrallazine injection $5 \mathrm{mg}$ every 30 minutes until the diastolic was less than $100 \mathrm{mmHg}$. Antihypertensive treatment was thereafter maintained with sublingual Nifedipine 20 mg. Continuous bladder drainage was instituted throughout the patients' hospital stay. The subsequent care and mode of delivery followed standard obstetric practice for pre-eclampsia/eclampsia.

\section{Data analysis}

At analysis, characteristics of participants with persistent hypertension and those who were normotensive at six weeks postpartum were compared. Baseline characteristics of the participants were compared using Pearson's chi-square test for categorical data and Student $t$-test for numerical data. To adjust for confounding and interaction, multivariate logistic-regression analysis was conducted to determine factors that were independently 
associated with the development of persistent hypertension, using STATA software. During modeling for regression analyses, all variables of clinical importance or with p-value 0.2 and less on bivariate analysis were considered for inclusion. Persistent hypertension entered as present $=1$, absent $=0$. Parity, number of living children and age of spouse were entered as numerical variables. Participant age was evaluated as a numerical variable and as 5-year age categories. The model goodness-of-fit of the final logistic regression models was assessed by Pearson's chi-square test.

\section{Ethical Considerations}

The study was approved by the research and ethics committees of Makerere University Faculty of Medicine and Mulago hospital. The participants gave written informed consent to participate in the study, whereby none were to be denied any healthcare in case they declined to participate or withdrew from the study. Medication and care for the pre-eclampsia/eclampsia were provided free to all participants.

\section{Results}

During the study period, 200 participants with preeclampsia/eclampsia were recruited into the study. We analyzed data for 195 participants: two participants died during follow up, while three failed to turn up for postpartum review in the postnatal clinic. Of the participants, 25 (12.8\%) had mild pre-eclampsia, 160 (82.1\%) had severe pre-eclampsia and 10 (5.1\%) had eclampsia. Only 150 patients (76.9\%) had full evaluation for HELLP syndrome, and of these, only 6 (3.1\%) had HELLP syndrome. Out of 195 participants, 54 (27.7\%) had persistent hypertension and 141(72.3\%) were found to have normal blood pressure at six weeks postpartum.

Table 1 shows the socio-demographic characteristics of the participants. Except for participants' age, there were no statistically significant differences in baseline characteristics of participants (between those who had persistent hypertension and those who were normotensive at six weeks postpartum) (p-value $>0.05$ ). Likewise, the difference in family history of hypertension, family history of diabetes, history of pre-eclampsia among siblings and lifestyle factors (smoking and alcohol drinking) was not statistically significant.

Table 2 compares the serum creatinine levels, seum uric acid levels, platelet count and urine protein levels at admission and after the puerperium among participants who had persistent hypertension and those who had become normotensive. Serum uric acid: the mean values of serum uric acid at admission were 417.7 (mg per $100 \mathrm{mls}$ ) versus 365.1 as compared to 317.6 versus 275.3 after six weeks postpartum. Although, at admission, the uric acid levels were higher among those who developed persistent hypertension, the difference between the two groups was not statistically significant. The difference in mean values of serum uric acid for those normotensive and those with persistent hypertension was statistically significant at 6 weeks postpartum.

Platelet count: for the platelet count, the mean values at both admission and six weeks postpartum were significantly higher in the group that had persistent hypertension.

Serum urea and creatinine: Though overall, the mean serum urea and creatinine levels were higher at admission and end of the puerperium in the group with persistent hypertension (as compared to the group who became normotensive), only the difference in mean levels at admission was statistically significant. Urine protein levels: Though urine protein levels at admission were comparable for the two groups (those who became normotensive and those with persistent hypertension) at recruitment, they were higher among those with persistent hypertension. For example, there was $9.3 \%$ with proteinuria of $3+$ among those with persistent hypertension compared to $2.9 \%$ among those normotensive after six weeks postpartum. Overall $44.4 \%$ of those with persistent hypertension had some degree of proteinuria, implying a strong association between postpartum proteinuria and persistent hypertension six weeks postpartum.

Table 3 shows the independent factors associated with persistence of hypertension. Participant age (especially among those in the category of 30-34 years) was statistically significant in predicting persistent hypertension. Serum creatinine at admission and urine protein at six weeks postpartum were significantly associated with persistent hypertension.

\section{Discussion}

The study assessed the incidence of persistent hypertension and factors associated with persistent hypertension in patients with pre-eclampsia/eclampsia. The study shows that the incidence of persistent hypertension is $27.7 \%$ (54/195). Participants' age (especially among women aged 30-34 years), serum creatinine at admission, serum uric acid six weeks postpartum and urine protein at six weeks postpartum were the factors independently associated with persistent hypertension. Family history of hypertension, history of diabetes and history of pre-eclampsia among siblings were not significantly associated with persistent hypertension.

The finding of higher mean serum uric acid levels and higher serum creatinine levels as well as urine protein in the women with persistent hypertension might result from renal dysfunction in pre-eclampsia. During normal pregnancy, the increased blood volume leads to marked reduction in serum uric acid and creatinine levels. Renal 
Table 1 Comparison of baseline characteristics of participants with and without persistent hypertension

\begin{tabular}{|c|c|c|c|c|c|}
\hline Characteristics & \multicolumn{2}{|c|}{$\begin{array}{l}\text { Normal Blood Pressure at } 6 \text { weeks postpartum } \\
\qquad n=141\end{array}$} & \multicolumn{2}{|c|}{$\begin{array}{l}\text { Persistent hypertension } 6 \text { weeks postpartum } \\
\qquad \begin{array}{r}n=54 \\
n \quad(\%)\end{array}\end{array}$} & P-value \\
\hline \multicolumn{6}{|l|}{ Age in complete years } \\
\hline $15-19$ & 36 & $(25.5)$ & 3 & (5.6) & \\
\hline $20-24$ & 49 & (34.6) & 18 & (33.3) & 0.001 \\
\hline $25-29$ & 35 & $(24.8)$ & 12 & $(22.2)$ & \\
\hline $30-34$ & 16 & $(11.3)$ & 13 & $(24.1)$ & \\
\hline $35-39$ & 3 & $(2.1)$ & 7 & $(12.6)$ & \\
\hline 40 years and above & 2 & $(1.4)$ & 1 & $(1.9)$ & \\
\hline \multicolumn{6}{|l|}{ Education } \\
\hline No formal education & 4 & $(2.9)$ & 1 & $(1.2)$ & \\
\hline Primary & 60 & $(42.9)$ & 21 & (39.6) & \\
\hline Secondary & 58 & $(41.4)$ & 29 & $(54.7)$ & 0.187 \\
\hline Tertiary & 18 & $(12.9)$ & 2 & (3.8) & \\
\hline \multicolumn{6}{|l|}{ Occupation } \\
\hline Peasant/house wife & 92 & $(65.2)$ & 35 & $(64.8)$ & \\
\hline Business woman & 35 & $(24.8)$ & 15 & $(27.8)$ & 0.325 \\
\hline Unemployed & 14 & $(9.9)$ & 4 & $(7.4)$ & \\
\hline \multicolumn{6}{|l|}{ Marital status } \\
\hline Single & 30 & $(21.3)$ & 8 & $(14.8)$ & \\
\hline Married & 111 & $(78.7)$ & 46 & $(85.2)$ & 0.640 \\
\hline \multicolumn{6}{|c|}{ Family history of Hypertension } \\
\hline Yes & 65 & $(46.1)$ & 32 & $(59.3)$ & \\
\hline No & 76 & 53.9) & 22 & $(40.7)$ & 0.230 \\
\hline \multicolumn{6}{|l|}{ History of Diabetes } \\
\hline Yes & 20 & $(14.2)$ & 11 & $(20.4)$ & \\
\hline No & 121 & $(85.8$ & 43 & $(79.6)$ & 0.570 \\
\hline \multicolumn{6}{|c|}{ History of pre-eclampsia among sisters } \\
\hline Yes & 26 & $(17.3)$ & 10 & $(19.2)$ & 0.752 \\
\hline No & 115 & $(82.7)$ & 42 & $(80.8)$ & \\
\hline \multicolumn{6}{|l|}{ History of smoking } \\
\hline Yes & 4 & $(2.8)$ & 2 & $(1.9)$ & 0.714 \\
\hline No & 137 & $(97.2)$ & 52 & $(98.1)$ & \\
\hline \multicolumn{6}{|l|}{ History of alcohol } \\
\hline Yes & 42 & $(29.8)$ & 18 & $(33.3)$ & 0.574 \\
\hline No & 99 & $(72.2)$ & 36 & $(66.6)$ & \\
\hline
\end{tabular}

blood flow and glomerular filtration rate are also markedly increased. In pre-eclampsia, renal perfusion and glomerular filtration are markedly reduced secondary to renal dysfunction. While the definite cause of renal dysfunction in pre-eclampsia remains unclear, one possible mechanism might be damage caused by endothelin, a potent endogenous vasoconstrictor peptide produced by endothelial cells [5]. Consequently, plasma uric acid levels are elevated in proportion to severity of preeclampsia. The renal insult secondary to vasospasm might be one of several potential factors involved in the pathogenesis of chronic hypertension among these women with pre-eclampsia. Likewise, chronic hypertension and pre-eclampsia may have similar or related risk factors. In women in whom endothelin levels are persistently elevated, serum uric acid is increased and creatinine clearance is diminished [5]. Elevated serum uric acid levels, which are prognostic for severe pre-eclampsia and eclampsia, are indicators of disease severity and remote prognosis for women at risk of renal and cardiovascular complications.

In this cohort study, there are several limitations. The blood pressure measurements beyond 6 weeks postpartum were only available for about half of the patients as many did not return for review beyond the six weeks. Data is not available on blood pressure at 12 weeks 
Table 2 Comparison of laboratory results (mean values) at admission and after 6 weeks for the two groups

\begin{tabular}{|c|c|c|c|}
\hline Variables Periods & $\begin{array}{l}\text { Normal BP Mean plus standard } \\
\text { deviation }\end{array}$ & $\begin{array}{l}\text { Abnormal BP (Persistent Hypertension) Mean plus } \\
\text { standard deviation }\end{array}$ & P-value \\
\hline + Serum uric acid $(\mu \mathrm{mol} / \mathrm{l})$ & $365.1 \pm 330.1$ & $417.7 \pm 200$ & 0.27 \\
\hline At admission & $275.3 \pm 200.6$ & $317.6 \pm 101.9$ & 0.002 \\
\hline \multicolumn{4}{|l|}{ After 6 weeks } \\
\hline + Mean Platelet level ( $\times 10^{9} /$ liter $)$ & $202.4 \pm 79.6$ & $228.9 \pm 96$ & 0.051 \\
\hline At admission & $241.9 \pm 59.2$ & $268.1 \pm 79$ & 0.014 \\
\hline \multicolumn{4}{|l|}{ After 6 weeks } \\
\hline + Serum Creatinine (mg/100 ml) & & $948 \pm 67.5$ & 0.004 \\
\hline At admission & $72.6 \pm 38.4$ & $251.7 \pm 1289$ & 0.071 \\
\hline After 6 weeks & $55.8 \pm 21.3$ & & \\
\hline + Serum Urea (mg/100 ml) & & $10.7 \pm 24.3$ & 0.066 \\
\hline At admission & $5.5 \pm 14.3$ & $8.6 \pm 17.9$ & 0.361 \\
\hline After 6 weeks & $6.5 .0 \pm 12.5$ & & \\
\hline \multicolumn{4}{|l|}{ *Urine protein at admission } \\
\hline+ & $1.0(0.7)$ & $0.0(0.0)$ & 0.165 \\
\hline++ & $60(42.6)$ & $22(40.7)$ & \\
\hline+++ & $57(40.4)$ & $16(29.6)$ & \\
\hline++++ & $23(16.3)$ & $16(29.6)$ & \\
\hline \multicolumn{4}{|c|}{ *Urine protein after 6 weeks postpartum } \\
\hline Negative & $104(74.3)$ & $26(48.2)$ & 0.000 \\
\hline Trace & $22(15.7)$ & $4(7.4)$ & \\
\hline+ & $8(5.7)$ & 16 (29.6) & \\
\hline ++ & $2(1.4)$ & $3(3.6)$ & \\
\hline+++ & $4(2.9)$ & $5(9.3)$ & \\
\hline
\end{tabular}

Key: *Figures indicate numbers and percentages of participants in both groups

†Figures indicate mean values and the standard deviation

Units for serum creatinine and urea are $\mathrm{mg}$ per $100 \mathrm{mls}$, while those for platelet count are $\left(\times 10^{9}\right.$ per liter.

Table 3 Predictors of persistent hypertension after 6 weeks

\begin{tabular}{llll}
\hline Predictors & Risk ratio & Confidence interval (CL) & P-value \\
\hline Age & 2.61 & $1.31-8.40$ & 0.027 \\
$15-19$ & 2.43 & $0.75-7.87$ & 0.138 \\
$20-24$ & 4.16 & $1.30-13.33$ & 0.017 \\
$25-29$ & 7.23 & $2.29-22.85$ & 0.001 \\
$30-34$ & 2.99 & $0.41-21.77$ & 0.278 \\
$35-39$ & 1.49 & $0.84-2.63$ & 0.172 \\
$40-44$ & & & 0.382 \\
Smoking & 1.79 & $0.48-6.67$ & 0.555 \\
Family history of hypertension & 0.87 & $0.55-1.38$ & 0.701 \\
History of alcohol & 0.92 & $0.58-1.47$ & 0.717 \\
History of pre-eclampsia & 1.06 & $0.67-1.67$ & 0.001 \\
Serum creatinine at admission & 2.04 & $1.35-3.09$ & 0.672 \\
Serum urea & 0.90 & $0.57-1.44$ & 0.051 \\
Urine protein at admission & 1.64 & $0.99-2.70$ & 0.035 \\
Serum uric acid at end of puerperium & 2.70 & $1.30-6.67$ & \\
\hline
\end{tabular}

postpartum. Secondly there limited data on HELLP syndrome as liver enzymes were not assessed routinely for all patients either at initiation of the cohort or during subsequent follow-up. Resolution of hypertension in pregnancy may occur beyond 6 weeks postpartum. Therefore, assessment at 6 weeks (or before 12 weeks postpartum) may over-estimate the incidence of persistent hypertension. We acknowledge this limitation as a possible explanation for the high incidence of persistent hypertension in this cohort. The incidence of persistent hypertension is rather high compared to that of Sibai et al. [2]. This difference could be attributable to the short 
period of 6 weeks of follow-up for our participants compared to Sibai's study of 7 years. While endothelial damage is the most likely factor in the pathogenesis of pre-eclampsia, many factors associated with pre-eclampsia as well as their mechanisms are not well understood.

Women who develop chronic hypertension following pre-eclampsia/eclampsia are often unaware of their problem and future risk. In a study to determine the blood pressure and renal function seven years after preeclampsia/eclampsia complicated pregnancy [6], only 10 out of 28 women with chronic hypertension knew they were hypertensive. Microalbuminuria in such women is common, is closely related to residual renal dysfunction and is an early marker of glomerular disease [6,7]. Women who develop recurrent second trimester severe pre-eclampsia or eclampsia are more likely to have an underlying renal disease and tend to have a high risk of developing chronic hypertension on follow up. In a study to establish the likelihood of an underlying renal disease or chronic hypertension among women with a history of pre-eclampsia, $10 \%$ developed chronic hypertension and $2 \%$ had an underlying renal disease [8]. This highlights the importance of follow up for all women managed for pre- eclampsia/eclampsia who have high serum uric acid and creatinine levels after puerperium. This enables identification of women with underlying renal disease or ongoing renal damage, both of which impact negatively on subsequent health and pregnancy outcome.

The finding that serum creatinine was a predictor for persistent hypertension implies that we could use baseline serum creatinine levels when the patients are admitted to predict women who are at risk of developing chronic hypertension in future. Whereas some studies doubt the prognostic value of uric acid for maternal and fetal outcome in hypertensive pregnancy $[9,10]$, hyperuricemia was associated with shorter gestations, lower birth weight, increased risk of preterm birth and risk of small-for-gestational-age infants, whether proteinuria was present or not [11]. This suggests that uric acid may be an important prognostic factor as effective as proteinuria at identifying gestational hypertensive pregnancies at increased risk [11].

Elevated uric acid levels in pre-eclamptic women may not simply be a marker of disease severity but possibly contribute directly to the pathogenesis of the disorder through ability of uric acid to promote inflammation, oxidative stress and endothelial dysfunction [12]. Increased adenosine may be a source of this uric acid [13]. Its levels are an indicator of ongoing oxidative stress [14] and could be responsible for the vascular injury of pre-eclampsia [15]. Though often considered an antioxidant, biochemical and in-vitro data indicate that noncrystalline, soluble uric acid can react to form radicals, increase lipid oxidation and induce various pro-oxidant effects in vascular cells [16]. From in-vitro and in-vivo studies, uric acid may contribute to endothelial dysfunction through inducing anti-proliferative effects on endothelium and impairing nitric oxide production in vascular smooth muscle cells (VSMCs) [16]. Hypertension develops through increased chemokine and cytokine expression, induction of the renin-angiotensin system and increased vascular C-reactive protein (CRP) expression [16]. This injury recovers within one month postpartum [17]. Improvement in glomerular filtration capacity is accompanied by recovery of hypertension to near-normal levels and significant improvement in albuminuria.

\section{Conclusion}

Nearly one of every four mothers with pre-eclampsia/ eclampsia are at risk of persistent hypertension at the end of the puerperium. Maternal age, serum creatinine and serum uric acid levels are predictors of persistent hypertension after the puerperium in women with preeclampsia and eclampsia.

\section{Acknowledgements}

The authors are grateful to colleagues of the Obstetrics and Gynecology Department of Makerere University, who gave invaluable advice on the design of the study, to the women who participated in the study and to the research assistants. Funding for this study came from Kulika Trust.

\section{Authors' contributions}

EBN was the graduate student who with DKK and MN as supervisors conceptualized and designed the study; carried out the data collection and follow up of the study participants, the data analysis and revised the draft manuscripts. MN contributed to design of the study, data analysis and interpretation and revision of the manuscripts. DKK was involved in the conception and design of the study, in the data analysis and interpretation, drafting of the initial manuscript and subsequent revision of the manuscripts. All co-authors approved the final manuscript.

\section{Competing interests}

The authors declare that they have no competing interests.

Received: 14 October 2009 Accepted: 12 March 2010

Published: 12 March 2010

\section{References}

1. Norwitz ER: Prevention of pre-eclampsia: is it possible? Clinical obstetrics and gynaecology 1999, 42:446-54

2. Sibai BM, Gonzalez-Ruiz A: Severe preeclampsia-eclampsia in young primigravid women: subsequent pregnancy outcome and remote prognosis. Am Journal Obstet Gynaecol 1986, 155:1011-6.

3. Sibai BM, Sarinoglu C: Severe preeclampsia in the second trimester: recurrence risk and long-term prognosis. Am J Obstet Gynaecol 1991 165:1408-1412.

4. Layla F, Badria ZA: Pre-eclampsia: is it a different disease in primiparous and multiparous women? Arch Gynecol Obstet 2005, 273:26-31.

5. Clark AB: Plasma endothelin levels in Pre-eclampsia: elevation and correlation with uric acid levels and renal impairment. Am J Obstet Gynecol 1992, 166:962-968.

6. Nisell $\mathrm{H}$ : Blood pressure and renal function seven years after pregnancy complicated by hypertension. BJOG 1995, 102:876-881.

7. North RA: What happens to women with pre-eclampsia? Microalbuminuria and hypertension following pre-eclampsia. Aust N Z J Obstet Gynecol 1996, 36:233-238. 
8. Reiter L: Hypertension in pregnancy: The incidence of underlying renal disease and essential hypertension. Am J Kidney Dis 1994, 24:883-887.

9. Paula $L G$, da Costa BE, Poli-de-Figueiredo CE, Antonello IC: Does uric acid provide information about maternal condition and fetal outcome in pregnant women with hypertension? Hypertens Pregnancy 2008, 27:413-20.

10. Williams KP, Galerneau F: The role of serum uric acid as a prognostic indicator of the severity of maternal and fetal complications in hypertensive pregnancies. J Obstet Gynaecol Can 2002, 24:628-632.

11. Roberts JM, Bodnar LM, Lain KY, Hubel CA, Markovic N, Ness RB, Powers RW: Uric acid is as important as proteinuria in identifying fetal risk in women with gestational hypertension. Hypertension 2005, 46:1263-1269.

12. Bainbridge $S A$, Roberts JM: Uric acid as a pathogenic factor in preeclampsia. Placenta 2008, 29:567-72.

13. Suzuki S, Yoneyama Y, Sawa R, Otsubo Y, Takeuchi T, Araki T: Relation between serum uric acid and plasma adenosine levels in women with preeclampsia. Gynecol Obstet Invest 2001, 51:169-72.

14. Tsukimori K, Yoshitomi T, Morokuma S, Fukushima K, Wake N: Serum uric acid levels correlate with plasma hydrogen peroxide and protein carbonyl levels in preeclampsia. Am J Hypertens 2008, 21:1343-1346.

15. Lam C, Lim KH, Kang DH, Karumanchi SA: Uric acid and pre-eclampsia. Semin Nephrol 2005, 25:56-60

16. Kanellis J, Kang DH: Uric acid as a mediator of endothelial dysfunction, inflammation, and vascular disease. Semin Nephrol 2005, 25:39-42.

17. Hladunewich MA, Myers BD, Derby GC, Blouch KL, Druzin ML, Deen WM, Naimark DM, Lafayette RA: Course of pre-eclamptic glomerular injury after delivery. Am J Physiol Renal Physiol 2008, 94:F614-620.

\section{Pre-publication history}

The pre-publication history for this paper can be accessed here:http://www. biomedcentral.com/1471-2393/10/12/prepub

doi:10.1186/1471-2393-10-12

Cite this article as: Ndayambagye et al.: Factors associated with persistent hypertension after puerperium among women with preeclampsia/eclampsia in Mulago hospital, Uganda. BMC Pregnancy and Childbirth 2010 10:12.

\section{Submit your next manuscript to BioMed Central and take full advantage of:}

- Convenient online submission

- Thorough peer review

- No space constraints or color figure charges

- Immediate publication on acceptance

- Inclusion in PubMed, CAS, Scopus and Google Scholar

- Research which is freely available for redistribution 